\title{
Towards flavored bound states beyond rainbows and ladders
}

\author{
B. El-Bennich*, E. Rojas*, M. A. Paracha*† and J. P. B. C. de Melo* \\ ${ }^{*}$ Laboratório de Física Teórica e Computacional, Universidade Cruzeiro do Sul, São Paulo 01506-000 SP, \\ Brazil \\ ${ }^{\dagger}$ Centre for Advanced Mathematics and Physics, National University of Science and Technology, Islamabad, \\ Pakistan
}

\begin{abstract}
We give a snapshot of recent progress in solving the Dyson-Schwinger equation with a beyond rainbow-ladder ansatz for the dressed quark-gluon vertex which includes ghost contributions. We discuss the motivations for this approach with regard to heavy-flavored bound states and form factors and briefly describe future steps to be taken.
\end{abstract}

Keywords: Mesons, Quarks \& Gluons, Nonperturbative QCD, Dyson-Schwinger equations, Bethe-Salpeter equations PACS: $11.10 . \mathrm{St}, 12.38 . \mathrm{Gc}, 12.38 . \mathrm{Lg}, 13.20 . \mathrm{He}, 14.40 . \mathrm{Lb}, 14.40 . \mathrm{Nd}, 14.65 . \mathrm{Bt}, 14.65 . \mathrm{Dw}, 14.65 . \mathrm{Fy}$

\section{MOTIVATION}

The challenge of understanding bound states in terms of the elementary fields of a given quantum field theory is persistent and of particular interest in Quantum Chromodynamics (QCD). The question of how precisely the quarks and gluons form hadrons immediately leads into the domain of relativistic quantum fields whose key properties can only be understood with nonperturbative methods. Paramount among the challenges is the understanding of confinement and dynamical chiral symmetry breaking (DCSB), both of which are likely to be intimately related [1].

Whereas certain simple two-body bound states and their resonances can be adequately described by potential models, this is not the case in QCD. Did light quarks not exist, the picture of string-like potentials arising from a flux tube between two (infinitely) heavy quarks would be correct. Yet, in the real world, where light current-quarks are ubiquitous, it is a feature of QCD that light-pair creation and annihilation effects are essentially nonperturbative and cannot be described by a quantum mechanical potential $[1,2]$. Such potentials are a poor guide to understanding the Goldstone boson of QCD and must be necessarily fine-tuned. In particular, in typical applications to hadronic form factors with "light" constituent quarks, the quark's propagation, $S(k)=\left(\gamma \cdot k-m_{q}\right)^{-1}$, is scale independent and does not describe confinement. In applications of relativistic quark models it was noted that this can lead to significant model dependance at larger momentum transfers $[3,4,5,6,7]$.

Heavy-light mesons are of additional interest since they exhibit some features of light-quark confinement. The important asymmetry in quark masses of flavor-nonsinglet $Q \bar{q}$ mesons leads to a disparate array of energy scales to be dealt with in solving the meson's relativistic bound-state equation. Thus, heavy mesons provide an excellent opportunity to study additional aspects of nonperturbative QCD and can be used to test simultaneously all manifestations of the Standard Model, namely the interplay between electroweak and strong interactions. Some of the major advances in heavy quark effective theory (HQET) [8] deal with factorization theorems allowing for a disentanglement of shortdistance or hard physics, which includes electroweak interactions and perturbative QCD (pQCD) contributions, from long-distance or soft physics, dominated by nonperturbative hadronic effects. The systematic reorganization of weak and QCD interactions in HQET has been treated in various approaches; e.g., with QCD factorization (QCDF) [9], pQCD [10] and soft-collinear effective theory (SCET) [11].

On the other hand, progress on nonperturbative matrix elements involving heavy-light states with flavor quantum numbers, $C= \pm 1$ and $B= \pm 1$, has been slower: while factorization theorems provide the means to systematically integrate out energy scales in the perturbative domain, valid in the infinitely heavy-quark limit, a reliable evaluation of the latter is notoriously difficult. Consider, for instance, the weak non-leptonic decay of a $B$ meson: $B \rightarrow M_{1} M_{2}$. If $M_{1}$ is a heavy or light(er) meson and $M_{2}$ a light meson [9], then the decay amplitude can be schematically written as,

$$
\left\langle M_{1} M_{2}\left|O_{i}\right| B\right\rangle=\left\langle M_{1}\left|j_{1}\right| B\right\rangle\left\langle M_{2}\left|j_{2}\right| 0\right\rangle\left[1+\sum_{n} r_{n} \alpha_{s}^{n}+\mathscr{O}\left(\Lambda_{\mathrm{QCD}} / m_{h}\right)\right]
$$


where $j_{1}$ and $j_{2}$ are the bilinear currents and $m_{h}$ is the heavy quark mass. The dimension-six effective four-quark operators, $O_{i}$, result from integrating out the weak gauge bosons $W^{ \pm}$in the operator product expansion. Multiplied by the appropriate Cabbibo-Kobayashi-Maskawa (CKM) matrix elements and Wilson coefficients, $C_{i}(\mu)$, which encode perturbative QCD effects above the renormalisation point $\mu$, the sum of these operators forms the heavyquark effective hamiltonian. Neglecting power corrections in $\alpha_{s}$ and taking the limit $m_{b} \rightarrow \infty$, the naive factorization is recovered. Higher orders in $\alpha_{s}$ break the factorization, yet in the limit $m_{h} \gg \Lambda_{\mathrm{QCD}}, \mathrm{pQCD}$ corrections beyond the naive factorization can systematically be accounted for. In the case of $B$ decays, the factorization, formally suppressed in $\Lambda_{\mathrm{QCD}} / m_{b}$, can be broken by weak annihilation decay amplitudes $[12,13]$ and final-state interactions between daughter hadrons $[14,15,16,17,18,19]$.

Moreover, since the charm quark is neither a light nor really a heavy quark, HQET may not be the adequate guide to charm physics and $\Lambda_{\mathrm{QCD}} / m_{c}$ corrections are significant [20,21]. Whilst effective Lagrangians based on approximate SU(4) flavor-spin symmetries ${ }^{1}$ are successful, see for example Refs. [22, 23, 24, 25], care should be taken when this symmetry is applied to the Lagrangian's effective couplings. As has been noted, $\mathrm{SU}(4)_{F}$ relations underestimate, for example, the $D \rho D$ coupling by a factor of four to five [26, 27]. In this context, it is also noteworthy that $\mathrm{SU}(3)_{F}$ and heavy-quark spin symmetry breaking effects are by no means insignificant and are manifest in the decay constant ratios, $f_{D_{s}} / f_{D}, f_{B_{s}} / f_{B}$ as well as $f_{D^{*}} / f_{D}$ and $f_{B^{*}} / f_{B}$; see, e.g., Section 4 in Ref. [21] and similar observations in lattice-QCD computations [28].

With respect to the weak decay constants, our focus in Eq. (1) is on the hadronic matrix elements, $\left\langle M_{1}\left|j_{1}\right| B\right\rangle$ and $\left\langle M_{2}\left|j_{2}\right| 0\right\rangle$. The latter represents the weak decay constant of $M_{2}$, which in the case of a pseudoscalar meson is given by,

$$
f_{M} P_{\mu}=\left\langle 0\left|\bar{q}_{a} \gamma_{\mu} \gamma_{5} q_{b}\right| M\right\rangle=Z_{2} \int \frac{d^{4} k}{(2 \pi)^{4}} \operatorname{tr}_{\mathrm{CD}}\left[\gamma_{5} \gamma_{\mu} S_{q}^{a}(k+\eta P) \Gamma_{M}^{a, b}(k ; P) S_{q}^{b}(k-\hat{\eta} P)\right],
$$

where $a, b$ collect flavor and color indices, $Z_{2}$ is the wave function renormalization constant, $S_{q}(k)$ are dressed quark propagators and $\Gamma(k ; P)$ is the mesons's Bethe-Salpeter amplitude (BSA). Note that in a Poincaré invariant treatment, the BSA and the weak decay constant — and any other hadronic matrix element — are independent of the momentum partitioning parameters $\eta+\hat{\eta}=1$. In some cases, for instance for the pseudoscalar mesons, $\pi, K, D, D_{s}$ and $B$, the values of the weak decay constants are known experimentally [29]. Much effort has also been invested to determine the heavy-light decay constants with lattice-regularized QCD, recently with unquenched fermions and increasingly better extrapolations to the continuum limit and physical pion masses [28, 30, 31, 32, 33, 34, 35]. Note that while the $c$-quark is treated as a propagating mode, the $b$-quark is usually implemented as a static fermion in lattice calculations of $f_{B}$ and $f_{B_{s}}$.

The first form factor in Eq. (1), $\left\langle M_{1}\left|j_{1}\right| B\right\rangle$, describes the transition of a heavy to a light(er) meson via the weak $V-A$ current and includes the propagation of the light spectator quark. Their precise evaluation is crucial in determining branching fractions and associated $C P$-violating observables of non-leptonic weak $D$ and $B$ decays $[14,16,17]$ and oscillations $[18,19]$. In semi-leptonic decays of heavy and light mesons they play a pivotal role in the determination of the Standard Model parameters, more precisely its weak sector via the CKM matrix elements. For example, the $K_{e 3}$ decay, $K \rightarrow \pi e v_{e}$, can be used to extract the matrix element $\left|V_{u s}\right| ; D_{e 3}$ to obtain $\left|V_{c s}\right|$; and the semi-leptonic decays of $B$ and $B_{c}$ mesons, in particular $B \rightarrow D \ell v_{\ell}, B_{c} \rightarrow D_{s}^{*} \ell^{+} \ell^{-}$and $B \rightarrow \pi \ell v_{\ell}$, inform the matrix elements $V_{c b}, V_{c s}$ and $V_{u b}$, respectively [36, 37]. As an example, we consider the case of heavy $H\left(0^{-+}\right)$to light(er) $P\left(0^{-+}\right)$transitions mediated by the weak HQET operators, $\bar{q}_{l} \gamma_{\mu}\left(1-\gamma_{5}\right) Q$, where the hadronic matrix element is completely described by two Lorentz vectors,

$$
\left\langle P\left(p_{2}\right)\left|\bar{q}_{l} \gamma_{\mu}\left(1-\gamma_{5}\right) Q\right| H\left(p_{1}\right)\right\rangle=F_{+}\left(q^{2}\right) P_{\mu}+F_{-}\left(q^{2}\right) q_{\mu},
$$

with the total heavy-meson momentum, $P_{\mu}=\left(p_{1}+p_{2}\right)_{\mu}, P^{2}=-M_{H}^{2}, q_{\mu}=\left(p_{1}-p_{2}\right)_{\mu}, Q=c, b$ and $q_{l}=u, d, s$.

Applications of light-quark propagators solutions of QCD's Dyson-Schwinger equations (DSE) in conjunction with the heavy-quark expansion to the form factors, $F_{+}\left(q^{2}\right)$ and $F_{-}\left(q^{2}\right)$, are in qualitative and quantitative agreement with heavy-quark symmetry [38, 39]. Yet, when the form factors in Eq. (3) are calculated both ways [38, 39], namely with the fully dressed heavy-quark propagator and the propagator in the heavy-quark limit, it is possible to verify the validity of said limit: corrections are of the order of $\simeq 20-30 \%$ are encountered in $b \rightarrow c$ transitions and can be as large as a factor of 2 in $c \rightarrow d$ transitions, as verified in a vast array of light- and heavy-meson observables [39].

\footnotetext{
1 i.e., models which implement $\mathrm{SU}(4)_{F}$ flavor symmetry in their Lagrangian approaches yet break these symmetries with the empirically known hadron masses.
} 
Moreover, the following ratios of transition form factors serve as a measure of $\mathrm{SU}(3)_{F}$ breaking:

$$
\frac{F_{+}^{B \rightarrow K}(0)}{F_{+}^{B \rightarrow \pi}(0)}=1.23, \quad \frac{A_{0}^{B \rightarrow K^{*}}(0)}{A_{0}^{B \rightarrow \rho}(0)}=1.25 .
$$

In Eq. (4), $A_{0}\left(q^{2}\right)$ are the appropriate form factors in $H\left(0^{-+}\right)$to $V\left(1^{--}\right)$transitions [39]. The flavor breaking is of similar order as for the decay constant ratios, $f_{D_{s}} / f_{D}$ and $f_{B_{s}} / f_{B}$, discussed in detail in Ref. [21].

For a summary of heavy-to-light transition form factor data from lattice-regularized QCD, see the review in Ref. [40]; we merely stress that contemporary lattice results are obtained for large squared-momentum transfer, i.e., $q^{2} \gtrsim 16 \mathrm{GeV}^{2}$ in the case of $B \rightarrow \pi$ transitions and $q^{2} \gtrsim 7 \mathrm{GeV}^{2}$ or $q_{\max }^{2}$ in $B \rightarrow D$ transitions $[41,42,43]$. Values at low $q^{2}$ must necessarily be extrapolated by means of appropriate parametrizations [44]. The rather strong quantitative differences between several predictions for the $B \rightarrow \pi$ form factor are emphasized in table 1 of Ref. [45] from which it is plain that model dependence is still the major obstacle to a precision calculation of even the simplest transition form factors.

In order to significantly improve on these form factor predictions in nonperturbative continuum QCD approaches, much progress has to be made in the hadronic description of heavy-light bound states. Within the framework of the DSEs and Bethe-Salpeter equations (BSE), recent efforts come to the conclusion that the so-called rainbow-ladder truncation fails to adequately reproduce basic static observables, such as the weak decay constant via Eq. (2) [46]. Other nonperturbative quantities are light-front distribution amplitudes which play an important role in QCDF analyses of hard exclusive processes. In particular, light-front projections of the pseudoscalar's BSA, namely the pseudoscalar and pseudotensor projections, are identified as twist-three two-particle distribution amplitudes for which estimations with QCD sum rules exist [47, 48, 49]. The pseudoscalar projection has recently been derived [50] and the same method may be applied to the pseudotensor projection. These projections can then be extended to $D$ and $B$ mesons provided a reliable BSA exists which allows for a faithful reproduction of experimental data on the respective weak decay constants. It is the aim of this contribution to sketch the path to a successful computation of the heavy-light meson's BSA.

\section{TWO, THREE, FOUR ... HOW MANY POINTS IN A MESON?}

In the continuum formulation, QCD's two-point Green functions are described by DSE, which provide the adequate nonperturbative approach. Likewise, mesons are quark-antiquark bound states which appear as poles in the 2-quark, 2-antiquark Green's function, $G^{(4)}=\left\langle 0\left|q_{1} q_{2} \bar{q}_{1} \bar{q}_{2}\right| 0\right\rangle$ These poles are found from studies of the inhomogeneous pseudoscalar and axialvector BSE [1, 51, 52, 53], as will be discussed shortly.

The Dyson or gap equation determines how quark propagation is influenced by interaction with the gauge fields. For a given quark flavor, the solutions of the quark DSE,

$$
S^{-1}(p)=Z_{2}\left(i \gamma \cdot p+m^{\mathrm{bm}}\right)+Z_{1} g^{2} \int_{k}^{\Lambda} \Delta^{\mu v}(q) \frac{\lambda^{a}}{2} \gamma_{\mu} S(k) \Gamma_{v}^{a}(-p, k, q),
$$

where $\int_{k}^{\Lambda} \equiv \int^{\Lambda} d^{4} k /(2 \pi)^{4}$ represents a Poincaré invariant regularization of the integral with the regularization mass scale, $\Lambda$, and $Z_{1,2}(\mu, \Lambda)$ are the vertex and quark wave-function renormalization constants. The (infinitely many) nonperturbative interactions alter the current-quark bare mass, $m^{\mathrm{bm}}(\Lambda)$, which receives corrections from the selfenergy given by the second term in Eq. (5), where the integral is over the dressed gluon propagator, $\Delta_{\mu \nu}(q)$, the dressed quark-gluon vertex, $\Gamma_{v}^{a}(-p, k, q)$, and $\lambda^{a}$ are the usual $\mathrm{SU}(3)$ color matrices of the fundamental representation. The gluon propagator is purely transversal in Landau gauge, which offers advantages in phenomenological interaction ansätze $[1,54]$ :

$$
\Delta_{\mu \nu}^{a b}(q)=\delta^{a b}\left(g_{\mu v}-\frac{q_{\mu} q_{v}}{q^{2}}\right) \Delta\left(q^{2}\right) .
$$

The quark-gluon vertex is given by $\Gamma_{\mu}^{a}\left(p_{1}, p_{2}, p_{3}\right)=g \frac{\lambda^{a}}{2} \Gamma_{\mu}\left(p_{1}, p_{2}, p_{3}\right)$ with the convention: $p_{1}+p_{2}+p_{3}=0$.

The solutions to the gap equation (5) are of the general form $S(p)=\left[i \gamma \cdot p A\left(p^{2}\right)+B\left(p^{2}\right)\right]^{-1}$ with the renormalization condition, $Z\left(p^{2}\right)=1 /\left.A\left(p^{2}\right)\right|_{p^{2}=\mu^{2}}=1$ at large spacelike $\mu^{2} \gg \Lambda_{\mathrm{QCD}}^{2}$. The mass function, $M\left(p^{2}\right)=$ $B\left(p^{2}, \mu^{2}\right) / A\left(p^{2}, \mu^{2}\right)$, is independent of the renormalization point $\mu$. In order to make quantitative matching with 
pQCD, another renormalization condition,

$$
\left.S^{-1}(p)\right|_{p^{2}=\mu^{2}}=i \gamma \cdot p+m(\mu)
$$

is imposed, where $m(\mu)$ is the renormalized running quark mass.

Before discussing Bethe-Salpeter amplitudes, we turn our attention to the quark-gluon vertex $\Gamma_{\mu}\left(p_{1}, p_{2}, p_{3}\right)$, which is one of QCD's three-point functions and satisfies its own BSE. In perturbation theory, that is for momenta $p_{1}^{2}=p_{2}^{2}=p_{3}^{2} \gtrsim \mu^{2}$, quark dressing effects are suppressed and $\Gamma_{\mu}\left(p_{1}, p_{2}, p_{3}\right) \rightarrow \gamma_{\mu}$. However, since the tremendous impact of DCSB on $Z\left(p^{2}\right)$ and $M\left(p^{2}\right)$ is nowadays well established, it is natural to accept that this also be true for the corresponding three-point functions.

In applications to hadron physics, practical models for the fermion-gauge boson vertex ought to satisfy fundamental symmetries of QCD. General ansätze to the nonperturbative vertex impose constraints of quantum field theory; as just mentioned, one insists that the vertex must reduce to the bare vertex $\gamma_{\mu}$ in the large-momentum limit (when dressed propagators can be replaced by bare propagators); it must have the same transformation properties as the bare vertex under charge conjugation $C$, parity transformation $P$ and time reversal $T$; it must ensure gauge covariance and invariance; and one demands that the vertex must be free of kinematic singularities. Finally, the full nonperturbative vertex can always be decomposed into a longitudinal and a transverse part, $\Gamma_{\mu}\left(p_{1}, p_{2}, p_{3}\right)=$ $\Gamma_{\mu}^{\mathrm{L}}\left(p_{1}, p_{2}, p_{3}\right)+\Gamma_{\mu}^{\mathrm{T}}\left(p_{1}, p_{2}, p_{3}\right)$ [55]. Clearly, gauge invariance is not satisfied for a bare vertex since it does not satisfy the Ward-Green-Takahashi identity (WGTI),

$$
i \gamma \cdot p_{3} \neq-i \gamma \cdot p_{1} A\left(p_{1}^{2}\right)+B\left(p_{1}^{2}\right)-i \gamma \cdot p_{2} A\left(p_{2}^{2}\right)-B\left(p_{2}^{2}\right) .
$$

Models that are largely consistent with the field theoretical constraints just mentioned have also been used to represent the dressed quark-gluon vertex, the most prominent amongst which is the Ball-Chiu ansatz for the longitudinal vertex [55]. However, while employed in studies of hadronic observables, the Ball-Chiu vertex satisfies a WGTI whereas the true quark-gluon vertex satisfies a Slavnov-Taylor identity (STI). The form of the latter, see Eq. (11), makes it plausible that within certain approximations a solution of

$$
p_{3 \mu} i \Gamma_{\mu}\left(p_{1}, p_{2}, p_{3}\right)=\mathscr{B}\left(p_{3}^{2}\right)\left[S^{-1}\left(-p_{1}\right)-S^{-1}\left(p_{2}\right)\right]
$$

can provide a reasonable approximation to the correct vertex.

In view of the scarce information on the quark-gluon vertex from first principle calculations, the strategy to combine different nonperturbative approaches to QCD was explored in Ref. [56]. Therein, lattice-QCD data for the dressedquark functions, $A\left(p^{2}\right)$ and $B\left(p^{2}\right)$ [57, 58], as well as for the gluon and ghost propagators, $\Delta\left(q^{2}\right)$ and $F\left(q^{2}\right)[59,60]$, were employed to numerically extract a momentum-dependent effective function $\tilde{X}_{0}\left(q^{2}\right)$ from the quark gap equation via an inversion procedure. In order to apply this inversion, one defines a "ghost-improved" Ball-Chiu vertex [61, 62],

$$
\tilde{\Gamma}_{\mu}^{\mathrm{BC}}\left(p_{1}, p_{2}, p_{3}\right)=\tilde{X}_{0}\left(p_{3}^{2}\right) F\left(p_{3}^{2}\right) \Gamma_{\mu}^{\mathrm{BC}}\left(p_{1}, p_{2}, p_{3}\right),
$$

which can be derived from the constraints of the STI,

$$
p_{3 \mu} i \Gamma_{\mu}\left(p_{1}, p_{2}, p_{3}\right)=F\left(p_{3}^{2}\right)\left[S^{-1}\left(-p_{1}\right) H\left(p_{1}, p_{2}, p_{3}\right)-\bar{H}\left(p_{2}, p_{1}, p_{3}\right) S^{-1}\left(p_{2}\right)\right],
$$

where $F\left(q^{2}\right)$ is the ghost-dressing function and the quark-ghost scattering kernel is parameterized in terms of the matrix-valued function, $H\left(p_{1}, p_{2}, p_{3}\right)$, and its conjugate, $\bar{H}\left(p_{1}, p_{2}, p_{3}\right)$ [63]. The decomposition of these two functions in terms of Lorentz covariants requires eight form factors,

$$
\begin{aligned}
& H\left(p_{1}, p_{2}, p_{3}\right)=X_{0} \mathbb{I}_{D}+i X_{1} \gamma \cdot p_{1}+i X_{2} \gamma \cdot p_{2}+i X_{3} \sigma_{\alpha \beta} p_{1}^{\alpha} p_{2}^{\beta}, \\
& \bar{H}\left(p_{2}, p_{1}, p_{3}\right)=\bar{X}_{0} \mathbb{I}_{D}-i \bar{X}_{2} \gamma \cdot p_{1}-i \bar{X}_{1} \gamma \cdot p_{2}+i \bar{X}_{3} \sigma_{\alpha \beta} p_{1}^{\alpha} p_{2}^{\beta} .
\end{aligned}
$$

Perturbative expressions for the form factors $X_{i}$ have been computed to one-loop order [63] and yield $X_{0}=1+\mathscr{O}\left(g^{2}\right)$ and $X_{i}=\mathscr{O}\left(g^{2}\right), i=1,2,3$. Thus, $X_{0}$ is the dominant form factor at large momenta and using the approximations $X_{1,2,3} \simeq 0$ and $\bar{X}_{0}=X_{0}=\tilde{X}_{0}\left(q^{2}\right)$ the dressed quark-gluon vertex reduces to the expression in Eq. (10) which satisfies the identity (9) with $\mathscr{B}\left(q^{2}\right)=\tilde{X}_{0}\left(q^{2}\right) F\left(q^{2}\right)$.

The quark-gluon vertex built from the resulting $\tilde{X}_{0}$ is enhanced in the infrared region and recovers the perturbative behavior as one approaches larger momenta. The two different inversion methods employed in Ref. [56], linear 

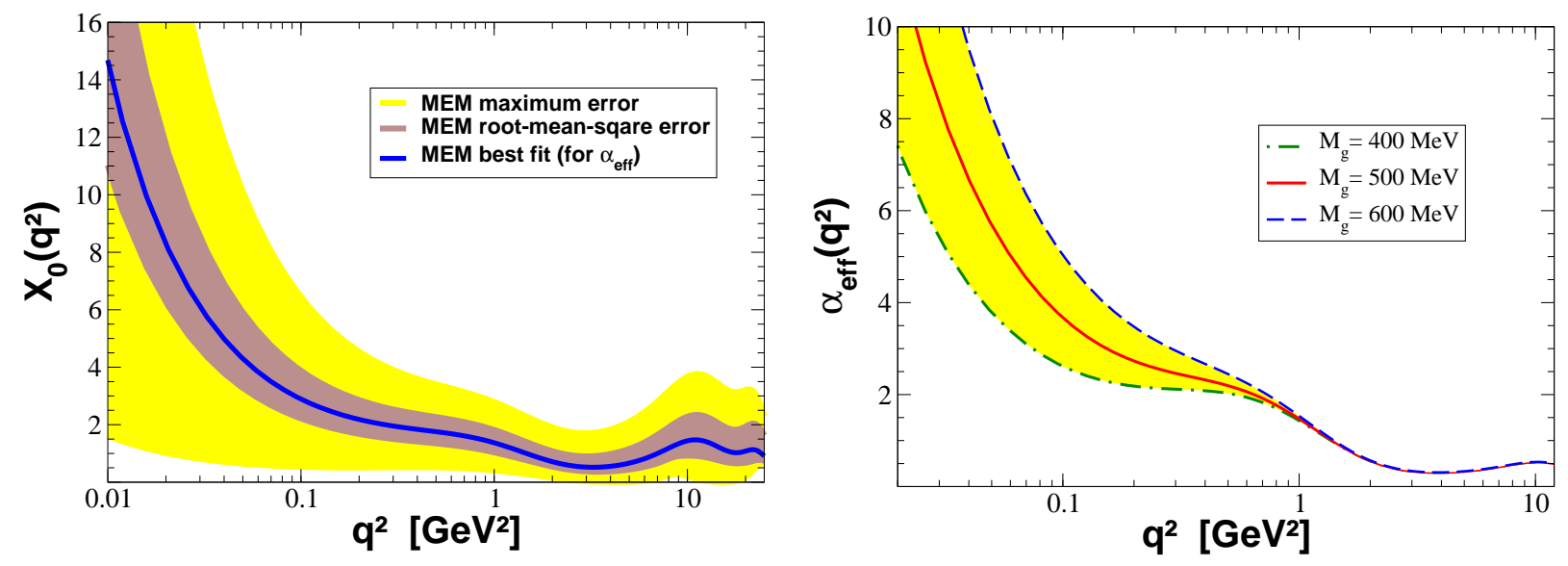

FIGURE 1. Left panel: the effective quark-gluon vertex function, $\tilde{X}_{0}^{\mathrm{MEM}}$, from a nonlinear inversion based on MEM; see Ref. [56] for details. Note that the functional form of $\tilde{X}_{0}^{\mathrm{MEM}}$ differs from that in Ref. [56] where $\tilde{X}_{0}^{\mathrm{MEM}}\left(q^{2} \rightarrow 0\right) \rightarrow 1$. Here it is clearly enhanced in the infrared due to the requirement that $q^{2} \tilde{X}_{0}\left(q^{2}\right)$ be finite in the MEM inversion. However, considering the case of maximal correlation of the MEM fit parameters, represented by the maximum error (yellow) band, the solution for $\tilde{X}_{0}$ in Ref. [56] is compatible with the present one. Owing to the lack of lattice-QCD data on $A\left(p^{2}\right)$ and $B\left(p^{2}\right)$, the inversion procedure is simply not constrained below $0.14 \mathrm{GeV}$. Nonetheless, either solution yields the mass functions in the left-hand plot of Fig. 2 since the DSE kernel is vanishing for $q \lesssim 0.2 \mathrm{GeV}$ and the functional form of $\tilde{X}_{0}$ thus becomes irrelevant in this momentum range. Right panel: the effective charge defined in Eq. (10) with $\tilde{X}_{0} \equiv \tilde{X}_{0}^{\mathrm{MEM}}$.

regularization and the maximum entropy method (MEM), produce $\tilde{X}_{0}$ form factors compatible with each other for the range of momenta where lattice-simulation data is available, i.e. in the domain $\simeq 0.3-4 \mathrm{GeV}$. For momenta in the range $0.3-1 \mathrm{GeV}$, both regularizations feature a strong yet somewhat different enhancement of the generalized Ball-Chiu vertex, as depicted in Fig. 1 for the MEM case, which generates the DCSB observed in the lattice-QCD mass functions [57, 58]. As can be read from the figure, for momentum values above $4 \mathrm{GeV}$, the extraction of $\tilde{X}_{0}\left(q^{2}\right)$ becomes less reliable owing to the lack of lattice-data constraints. Similarly, the steep increase of $\tilde{X}_{0}\left(q^{2}\right)$ below $\simeq 0.1 \mathrm{GeV}^{2}$ is to be taken with caution. Nonetheless, the functional form of $\tilde{X}_{0}$ above $q^{2} \gtrsim 0.1 \mathrm{GeV}^{2}$ bears strong similarities with that of the ghost-dressing function [59,60], $F\left(q^{2}\right)$, which a posteriori justifies the prescription $F\left(q^{2}\right) \rightarrow F^{2}\left(q^{2}\right)$ employed in Ref. [61]. We thus, in analogy with Ref. [64], define an effective charge via the combination,

$$
\alpha_{\mathrm{eff}}\left(q^{2}\right)=\alpha_{s} \tilde{X}_{0}\left(q^{2}\right) F\left(q^{2}\right) \Delta\left(q^{2}\right)\left[q^{2}+m_{g}^{2}\left(q^{2}\right)\right] ; \quad m_{g}^{2}\left(q^{2}\right)=\frac{M_{g}^{4}}{q^{2}+M_{g}^{2}},
$$

plotted in Fig. 1, where $\alpha_{s}(4.3 \mathrm{GeV})=0.295$ and $M_{g}^{2}$ typically of the order $500-600 \mathrm{MeV}$.
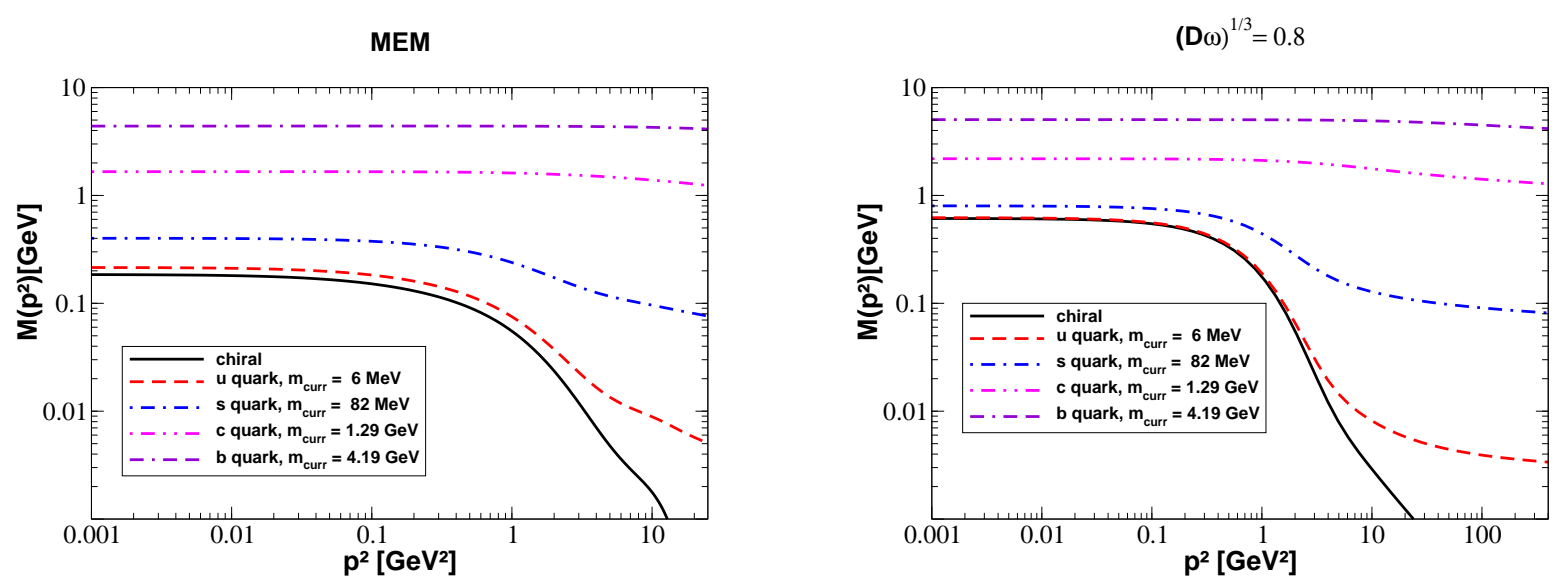

FIGURE 2. Flavor dependence of the solutions for the mass function $M\left(p^{2}\right)=B\left(p^{2}\right) / A\left(p^{2}\right)$; left panel: using the effective interaction model of Eq. (14); right panel: DSE with interaction model and given parameter set of Ref. [65]. 

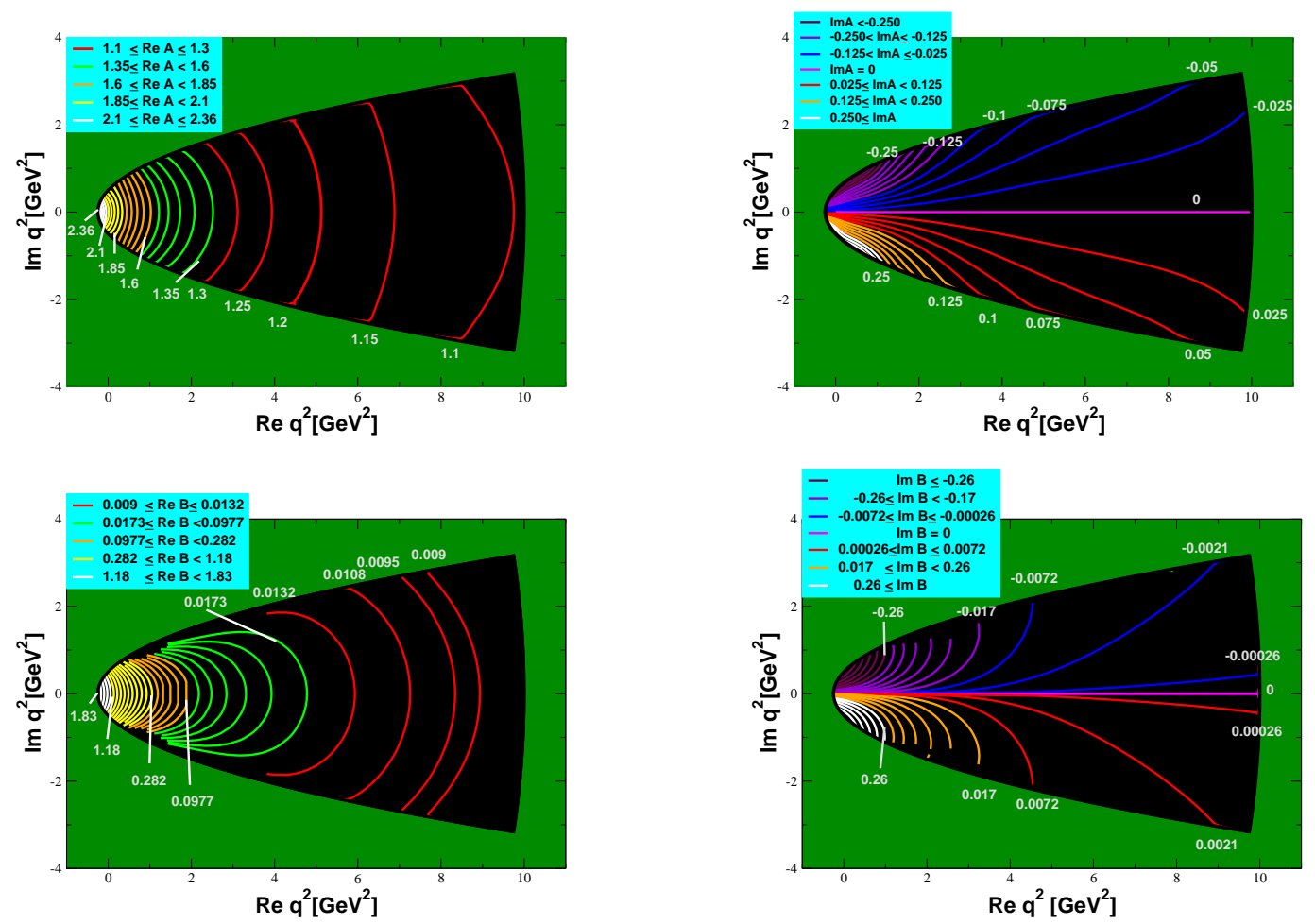

FIGURE 3. Contour levels of the real and imaginary parts of the DSE solutions, $A\left(p^{2}\right)$ and $B\left(p^{2}\right)$, for complex momenta using the interaction model of Ref. [65].

The flavor dependence of the solutions for the mass function is depicted in the left panel of Fig. 2. For the light quarks, $u$ and $d$, the DCSB leads to $M(0) \simeq 220 \mathrm{MeV}$ in accordance with lattice results [57, 58]. Yet, in comparison with the best available phenomenological interaction model [65] whose functional behavior accords qualitatively with results of modern DSE and lattice studies, the effect of the DCSB is much weaker: as seen in the right panel of Fig. 2, the model of Ref. [65] yields $M(0) \simeq 600 \mathrm{MeV}$. Although the consequences of DCSB are less marked for the heavy quarks' mass functions, which remain almost constant over a large momentum domain, we do note a difference $\Delta M_{b}(0) \simeq 600 \mathrm{MeV}$ between both models. It is thus expected that the interaction defined by Eq. (14) is too weak for applications to hadron phenomenology. This shortcoming may be remedied by the inclusion of the transverse quark-gluon vertex component which describes the quark's anomalous chromomagnetic moment [66].

Mass functions are not physical observables and to test the validity and efficacy of an interaction model it must stand the comparison with experimental data. To this end, bound state equations must be solved and while the propagator satisfies the gap equation, the vertices are determined by an inhomogeneous BSE. Consider, for instance, the exact inhomogeneous axialvector BSE [67] which is valid when the quark-gluon vertex is fully dressed, i.e. for an ansatz beyond the rainbow-ladder truncation:

$$
\begin{aligned}
\Gamma_{5 \mu}^{f g}(k ; P) & =Z_{2} \gamma_{5} \gamma_{\mu}-g^{2} \int_{q}^{\Lambda} D^{\alpha \beta}(k-q) \frac{\lambda^{a}}{2} \gamma_{\alpha} S_{f}\left(q_{+}\right) \Gamma_{5 \mu}^{f g}(q ; P) S_{g}\left(q_{-}\right) \frac{\lambda^{a}}{2} \Gamma_{\beta}^{g}\left(q_{-}, k_{-}\right) \\
& +g^{2} \int_{q}^{\Lambda} D^{\alpha \beta}(k-q) \frac{\lambda^{a}}{2} \gamma_{\alpha} S_{f}\left(q_{+}\right) \frac{\lambda^{a}}{2} \Lambda_{5 \mu \beta}^{f g}(k, q ; P),
\end{aligned}
$$

where $P$ is the total meson momentum, $q_{ \pm}=q \pm P / 2, k_{ \pm}=k \pm P / 2$ and $f, g$ denote the flavor indices of a light-light or heavy-light bound state. The 4-point Schwinger function $\Lambda_{5 \mu \beta}^{f g}$ is entirely defined via the quark self energy. This comes about that a WGTI can be derived for the Bethe-Salpeter kernel whose solution provides a symmetry-preserving closed system of gap and vertex equations $[67,68]$. The pseudoscalar vertex, $\Gamma_{5}^{f g}(k ; P)$, satisfies an analogous equation to Eq. (15) and it is a well known feature of QCD that both the axialvector and pseudoscalar vertices exhibit poles 
whenever $P^{2}=-m_{M_{n}}^{2}$, where $m_{M_{n}}$ is the mass of the meson $M$ or any of its radial excitations $[69,70]$ :

$$
\left.\Gamma_{5 \mu}(k ; P)\right|_{P^{2}+m_{M_{n}}^{2} \simeq 0}=\frac{f_{M_{n}} P_{\mu}}{P^{2}+m_{M_{n}}^{2}} \Gamma_{M_{n}}(k ; P)+\Gamma_{5 \mu}^{\mathrm{reg} .}(k ; P),
$$

Here, $\Gamma_{M_{n}}(k ; P)$ is the pseudoscalar bound state's BSA. The solutions of the BSE for $P^{2}=-m_{M_{n}}^{2}$ in Euclidean momentum space requires the knowledge of the quark propagator at complex momenta whose squares lie inside a parabola and which in the past presented a considerable numerical challenge at large quark masses, $m_{q}>m_{c}$. Improved numerical methods which facilitate the treatment of the quark's DSE are now available [71] and in Fig. 3 we present the real and imaginary parts of the complex solutions $A\left(p^{2}\right)$ and $B\left(p^{2}\right)$, where $P^{2} \simeq-1 \mathrm{GeV}^{2}$. Solutions for heavier quarks are currently being investigated and in conjunction with Eq. (15) first results for the heavy meson's BSA using Eq. (10) will soon be available.

\section{EPILOGUE}

We have summarized recent progress towards computing the DSE and BSE for heavy-light systems beyond the rainbow-ladder truncation based on an ansatz for the quark-gluon vertex which correlates the tensor structure of the Ball-Chiu vertex with a nonperturbative vertex function. The functional form of the latter is extracted from latticeregulated QCD data on the quark's dressed propagator via an inversion of the DSE [56]. When the vertex model is re-inserted in the quark's DSE, its solutions yield mass functions which are qualitatively comparable with those obtained with a recent interaction model [66] but whose magnitude of DCSB is considerably smaller. Computations of the BSA for heavy-light systems which make use of the exact form of the BSE, valid for the fully dressed quarkgluon vertex, and either interaction models are underway. The first test any beyond the rainbow-ladder ansatz for the quark-gluon vertex must pass is the numerical value one obtains for one of the most elementary observable, i.e. the weak decay constant which is more sensitive to the BSA normalization. We shall report results of its computation for $D_{(s)}$ and $B_{(s)}$ mesons in a future communication. This will be the first in a series of steps to obtain their form factors and parton distribution amplitudes that are our original motivation (see Section 1) and for which the well known rainbow-ladder ansätze $[69,70]$ are not phenomenologically valuable.

\section{ACKNOWLEDGMENTS}

This work is supported by the São Paulo Research Foundation, Fundação de Amparo à Pesquisa do Estado de São Paulo (FAPESP), and the federal agency, Conselho Nacional de Desenvolvimento Científico e Tecnológico (CNPq). We acknowledge valuable communication with Orlando Oliveira and Tobias Frederico. B. E. would like to thank the organizers of the XXXVI Reunião de Trabalho sobre Física Nuclear no Brasil for their kind support.

\section{REFERENCES}

1. A. Bashir, L. Chang, I. C. Cloët, B. El-Bennich, Y.-x. Liu, C. D. Roberts and P. C. Tandy, Commun. Theor. Phys. 58, 79 (2012).

2. L. Chang, I. C. Cloët, B. El-Bennich, T. Klähn and C. D. Roberts, Chin. Phys. C 33, 1189 (2009).

3. B. El-Bennich, J. P. B. C. de Melo, B. Loiseau, J.-P. Dedonder and T. Frederico, Braz. J. Phys. 38, 465 (2008).

4. B. El-Bennich, O. Leitner, J.-P. Dedonder and B. Loiseau, Phys. Rev. D 79, 076004 (2009).

5. E. O. da Silva, J. P. B. C. de Melo, B. El-Bennich and V. S. Filho, Phys. Rev. C 86, 038202 (2012).

6. B. El-Bennich, J. P. B. C. de Melo and T. Frederico, Few Body Syst. 54 (2013) 1851.

7. J. P. B. C. de Melo, T. Frederico, E. Pace, and G. Salmè, Phys. Rev. D 73, 074013 (2006).

8. G. Buchalla, A. J. Buras and M. E. Lautenbacher, Rev. Mod. Phys. 68, 1125 (1996).

9. M. Beneke, G. Buchalla, M. Neubert and C. T. Sachrajda, Phys. Rev. Lett. 83, 1914 (1999); id., Nucl. Phys. B 591, 313 (2000).

10. Y. Y. Keum, H. N. Li and A. I. Sanda, Phys. Rev. D 63, 054008 (2001).

11. C. W. Bauer et al., Phys. Rev. D 63, 114020 (2001); C. W. Bauer, D. Pirjol and I. W. Stewart, ibid. 65, 054022 (2002);

C. W. Bauer et al., ibid. 66, 014017 (2002).

12. C. M. Zanetti and A. A. Natale, arXiv:1007.5072 [hep-ph] (2010).

13. A. A. Natale and C. M. Zanetti, Int. J. Mod. Phys. A 24, 4133 (2009).

14. B. El-Bennich A. Furman, R. Kamiński, L. Leśniak and B. Loiseau, Phys. Rev. D 74, 114009 (2006).

15. D. R. Boito, B. El-Bennich, B. Loiseau and O. Leitner, Int. J. Mod. Phys. E 16, 2876 (2007). 
16. D. R. Boito, J.-P. Dedonder, B. El-Bennich, O. Leitner and B. Loiseau, Phys. Rev. D 79, 034020 (2009).

17. B. El-Bennich, A. Furman, R. Kamiński, L. Leśniak, B. Loiseau and B. Moussallam, Phys. Rev. D 79, 094005 (2009). [Erratum-ibid. D 83, 039903 (2011)]

18. O. Leitner, J.-P. Dedonder, B. Loiseau and B. El-Bennich, Phys. Rev. D 82, 076006 (2010).

19. B. El-Bennich, J. P. B. C. de Melo, O. Leitner, B. Loiseau and J. P. Dedonder, Prog. Part. Nucl. Phys. 67, 395 (2012).

20. B. El-Bennich, M. A. Ivanov and C. D. Roberts, Phys. Rev. C 83, 025205 (2011)

21. B. El-Bennich, C. D. Roberts and M. A. Ivanov, POS(QCD-TNT-II)018, arXiv:1202.0454 [nucl-th] (2012).

22. C. W. Xiao, J. Nieves and E. Oset, Phys. Rev. D 88, 056012 (2013).

23. R. Molina, H. Nagahiro, A. Hosaka and E. Oset, Phys. Rev. D 80, 014025 (2009).

24. G. Krein, A. W. Thomas and K. Tsushima, Phys. Lett. B 697, 136 (2011).

25. K. Tsushima, D. H. Lu, G. Krein and A. W. Thomas, Phys. Rev. C 83, 065208 (2011).

26. B. El-Bennich, G. Krein, L. Chang, C. D. Roberts and D. J. Wilson, Phys. Rev. D 85, 031502 (2012).

27. K. U. Can, G. Erkol, M. Oka, A. Ozpineci and T. T. Takahashi, Phys. Lett. B 719, 103 (2013).

28. D. Bečirević, V. Lubicz, F. Sanfilippo, S. Simula and C. Tarantino, arXiv:1201.4039 [hep-lat].

29. J. Beringer et al. [Particle Data Group Collaboration], Phys. Rev. D 86, 010001 (2012).

30. P. Dimopoulos et al. [ETM Collaboration], JHEP 1201, 046 (2012).

31. H. Na, C. T. H. Davies, E. Follana, G. P. Lepage and J. Shigemitsu, Phys. Rev. D 86, 054510 (2012).

32. A. Bazavov et al. [Fermilab Lattice and MILC Collaborations], Phys. Rev. D 85, 114506 (2012).

33. Y. Namekawa et al. [PACS-CS Collaboration], Phys. Rev. D 84, 074505 (2011).

34. A. Bazavov et al. [Fermilab Lattice and MILC Collaborations], PoS LATTICE 2012, 159 (2012).

35. N. Carrasco et al., arXiv:1308.1851 [hep-lat].

36. M. A. Ivanov, Yu. L. Kalinovsky, P. Maris and C. D. Roberts, Phys. Lett. B 416, 29-35 (1998).

37. M. Ali Paracha, Ishtiaq Ahmed and M. Jamil Aslam, Phys. Rev. D 84, 035003 (2011).

38. M. A. Ivanov, Yu. L. Kalinovsky and C. D. Roberts, Phys. Rev. D 60, 034018 (1999).

39. M. A. Ivanov, J. G. Körner, S. G. Kovalenko and C. D. Roberts, Phys. Rev. D 76, 034018 (2007).

40. S. Aoki et al., arXiv:1310.8555 [hep-lat].

41. G. M. de Divitiis, E. Molinaro, R. Petronzio and N. Tantalo, Phys. Lett. B 655, 45 (2007).

42. J. A. Bailey et al. (Fermilab Lattice and MILC Collaborations), Phys. Rev. D 85, 114502 (2012) [Erratum-ibid. D 86, 039904 (2012)].

43. M. Atoui, D. Bečirević, V. Morenas and F. Sanfilippo, arXiv:1310.5238 [hep-lat].

44. D. Bečirević and A. B. Kaidalov, Phys. Lett. B 478, 417 (2000).

45. B. El-Bennich, M. A. Ivanov and C. D. Roberts, Nucl. Phys. Proc. Suppl. 199, 184 (2010).

46. T. Nguyen, N. A. Souchlas and P. C. Tandy, AIP Conf. Proc. 1361, 142 (2011).

47. V. M. Braun and I. E. Filyanov, Z. Phys. C 48, 239 (1990) [Sov. J. Nucl. Phys. 52, 126 (1990)] [Yad. Fiz. 52, 199 (1990)].

48. P. Ball, JHEP 9901, 010 (1999)

49. P. Ball, V. M. Braun and A. Lenz, JHEP 0605, 004 (2006).

50. L. Chang, C. D. Roberts and S. M. Schmidt, arXiv:1308.4708 [nucl-th].

51. C. D. Roberts, Prog. Part. Nucl. Phys. 61, 50 (2008).

52. P. Maris and P. C. Tandy, Nucl. Phys. Proc. Suppl. 161, 136 (2006).

53. A. Krassnigg and C. D. Roberts, Fizika B 13, 143 (2004).

54. K. Raya, A. Bashir, S. Hernández-Ortiz, A. Raya and C. D. Roberts, arXiv:1305.2955 [nucl-th].

55. J. S. Ball and T.-W. Chiu, Phys. Rev. D 22, 2542 (1980).

56. E. Rojas, J. P. B. C. de Melo, B. El-Bennich, O. Oliveira and T. Frederico, in press, JHEP; arXiv:1306.3022 [hep-ph].

57. M. B. Parappilly et al., Phys. Rev. D 73, 054504 (2006).

58. S. Furui and H. Nakajima, Phys. Rev. D 73, 074503 (2006).

59. I. L. Bogolubsky, E. M. Ilgenfritz, M. Müller-Preussker and A. Sternbeck, Phys. Lett. B 676,69 (2009).

60. O. Oliveira and P. J. Silva, Phys. Rev. D 86, 114513 (2012).

61. A. C. Aguilar and J. Papavassiliou, Phys. Rev. D 83, 014013 (2011).

62. C. S. Fischer and R. Alkofer, Phys. Rev. D 67, 094020 (2003).

63. A. I. Davydychev, P. Osland and L. Saks, Phys. Rev. D 63, 014022 (2001).

64. A. Ayala, A. Bashir, D. Binosi, M. Cristoforetti and J. Rodríguez-Quintero, Phys. Rev. D 86, 074512 (2012).

65. S.-x. Qin, L. Chang, Y.-x. Liu, C. D. Roberts and D. J. Wilson, Phys. Rev. C 84, 042202 (2011).

66. L. Chang, Y. -X. Liu and C. D. Roberts, Phys. Rev. Lett. 106, 072001 (2011).

67. L. Chang and C. D. Roberts, Phys. Rev. Lett. 103, 081601 (2009).

68. L. Chang and C. D. Roberts, Phys. Rev. C 85, 052201 (2012).

69. P. Maris, C. D. Roberts and P. C. Tandy, Phys. Lett. B 420, 267 (1998).

70. P. Maris and C. D. Roberts, Phys. Rev. C 56, 3369 (1997).

71. A. Krassnigg, PoS CONFINEMENT 8, 075 (2008) [arXiv:0812.3073 [nucl-th]]. 